\title{
Trace Elements Profile among Alcohol Abusers in a Nigerian Community ${ }^{1}$ STANLEY, P C; ${ }^{2}$ OKEKE, E, ${ }^{2}$ UKOLI, C
}

\author{
${ }^{1}$ Department of Medicine, Mental Health Unit, University of Port Harcourt Teaching Hospital (UPTH) \\ P.M.B. 6173, Port Harcourt, Nigeria \\ E-mail: stanleypc2001@yahoo.com \\ ${ }^{2}$ Jos University Teaching Hospital (JUTH), Jos
}

\begin{abstract}
Alcohol abuse has been associated with Trace elements deficiencies which have been known to cause Neuro Psychiatric Disorders. The focus of this study being on locally brewed alcohol is to establish the pattern if any of trace elements derangements. The trace element profile among alcohol abusers in a Nigerian Community was analysed using the 4 - item CAGE instrument to separate alcohol abusers, social drinkers, and total abstainers. The Royal College of Physicians' criteria 1987 for Alcohol abuse was also used to validate the CAGE score. Using the CAGE score, the following results were obtained, 50 Alcohol abusers (AOA), 47 Social Drinkers (SOD) and 45 Total Abstainers (TOA) emerged out of 162 subjects. Mean values obtained for Manganese (Mn), Zinc ( $\mathrm{Zn})$, Iron $(\mathrm{Fe})$ and Copper $(\mathrm{Cu})$ were $0.17 \mathrm{nmol} / \mathrm{L}, 9.03 \mathrm{umol} / \mathrm{L}, 27.46 \mathrm{umol} / \mathrm{L}$ and 25.44 umol/L respectively. This showed that $\mathrm{Mn}, \mathrm{Fe}$ and $\mathrm{Cu}$ were significantly increased while $\mathrm{Zn}$ was reduced significantly in alcohol abusers when compared with the control group of total abstainers. Excessive consumption of burukutu seems to significantly alter the trace elements status in abusers. Duration of intake and excessive use of burukutu can be associated with both deficiency and toxicity of essential and heavy metals. Abstinence therefore should continue to be the main stay of counseling. @JASEM
\end{abstract}

The functions of trace elements and other abundant metals are determined in part by their charges, mobilities and binding constants to biological ligands. These elements play an important role in the formation and maintenance of the internal environment by which the human nervous system responds to external stimuli. Some of these elements in minute quantities are essential in the metabolic process of neurotransmitters (Srinivasan 1984). Chronic alcohol abuse has been associated with deficiencies of such essential trace elements like iron and zinc. This was further attributed to dietary caused association with under nutrition and mal-absorption commonly reported among alcoholics \{Sanderson, 1978; Prasad, 1979; Schukit, 1998).

Nuero psychiatric disorders may arise either from trace elements deficiencies or toxicity. Deficiencies of zinc and iron have been associated with various forms of neuropsychiatric disorders. High Serum levels of copper and manganese have been associated with psychotic depression and Bipolar Affective Disorders (Stanley, PC and Wakwe VC 2000). Furthermore, hypertension has been reported among progressive alcohol abusers with rising levels of heavy metals in their sera \{Pfeiffer; Mailoux, 1988). Biomedical research has, however, indicated the presence of variations in the biological response of different ethnic groups of alcohol consumption attributed mainly to genetic differences $\{$ Fenna et al., 1971)\}. In consideration of this noted racial differences and the paucity of studies on the trace elements status of alcohol users among Nigerians, this study was designed to investigate the trace element profile among alcohol abusers in the Kugiya Community, of Jos Plateau in Nigeria.

\section{MATERIALS AND METHODS}

This study was community and laboratory based. It was conducted among the Kugiya people, an entirely indigenous population in Jos suburb, Plateau State of
Nigeria with a population of about 3,800 people whose major occupation is farming and brewing of a local alcohol, burukutu from grains, usually guinea corn by fermentation for local consumption. It is predominantly a Christian ethnic group.

There were 1,202 adults of ages 16 years and above with a total number of 320 families. Out of these 320 families, adults of 16 - 54 years of age were selected, one from every two families and a total of 160 subjects emerged. They were further subjected to screening using 4-item CAGE instrument \{Mayfield et al., 1974)\} and quantity of alcohol taken using the criteria of the Royal College of Physician (RCP 1987) for alcohol abuse. Based on these criteria the subjects were divided into alcohol abuser (AOA), social drinkers (SOD) and total abstainers (TOA). They were age and sex matched and consents for the study was obtained from the Chief of the community, the subjects and their relations. Pregnant woman, chronically ill and those with fever were excluded from the study. Ten millilitres $(\mathrm{ml})$ of venous blood was collected from each subject. The samples were centrifuged within 12 hours of collection and the sera stored in the freezer for the analysis of manganese, zinc, iron and copper within a week from the date of collection.

Ethical standards of the Ethnic Committee of Jos University Teaching Hospital (JUTH) were observed throughout this study. The data were analysed by computer using the SPSS programme. Statistical methods applied included: 1. Cross tabulations, 2. Chi-square (with Yates correction where necessary), 3. Analysis of Variance (ANOVA) for multiple comparisons of mean.

\section{RESULTS AND DISCUSSION}

The mean age of the subjects was 33 years for alcohol abusers and 35 years for social drinkers. The mean duration of intake was 12.8 years for alcohol abusers and 10.2 years 
for social drinkers. The mean quantity taken were 48.2 units and 1.3 units for alcohol abusers and social drinkers respectively. Ten percent of alcohol abusers took predominantly alcohol (beer and wine), $60 \%$ took burukutu predominantly and $30 \%$ took burukutu and industrial alcohol significantly. There were significant differences $(\mathrm{P}<0.001)$ between mean duration and quality of alcohol taken between the alcoholics and social drinkers. All the values obtained from total abstainers were in the normal range for these trace elements. The following were obtained for alcohol abusers and social drinkers: Manganese was high $(0.17 \mathrm{nmol} / \mathrm{L}$ in AOA when compared with $0.09 \mathrm{nmol} / \mathrm{L}$ in the TOA. The values obtained from the social drinkers were within the normal range. Zinc was reduced in AOA $(9.03 \mathrm{umol} / \mathrm{L}$ but within normal limits for SOD and TOA. Iron was raised $(27.46 \mathrm{umol} / \mathrm{L}$ in $\mathrm{AOA}$ than in SOD $21.76 \mathrm{umol} / \mathrm{L}$ ) compared with $16.57 \mathrm{nmol} / \mathrm{L}$ in TOA. All the alternations in trace elements were significant.

The predominantly higher levels of copper and iron amongst alcohol abusers who took only burukutu agrees with the finding of Okoye et al (1984). The presence of such elements being associated with the process of brewing. The rusty drums, iron pots and lead pipes used in stirring are the likely sources of these elements. Hence, the reported higher serum iron among alcohol abusers in this study disagrees with the reported findings of low iron levels among alcohol abusers (Sanderson,1978; Schukit, 1998)

Serum copper and manganese levels increased from social drinkers through alcohol abusers. This finding is similar to that of Cawte and Florence (1989)\}.

This may be indicative of progressive alcohol toxicity. Furthermore, the changes in trace elements could be due to malnutrition, malabsorption and diuresis associated with chronic alcoholic abuse (Sanderson,1978; Schukit, 1998).

There were hypoproteinaemia and hypoalbuminaemia. The low protein levels may explain. The low levels of serum zinc but not an accompanying increase in serum copper, manganese and iron, which also bind to these proteins. The reported low serum levels of zinc agrees with the findings of other workers (Sanderson,1978 Prasad, 1979).

Toxic amounts of these elements are associated with different disease conditions. Exposure to large amounts of manganese can be neuro toxic, producing a dyskinetic motor syndrome similar to Parkinson's disease (Chu et al 1995). Zinc associated with gastric ulcer, pancreatitis, lethargy, anemia, fever, nausea, vomiting, respiratory distress and pulmonary fibrosis. Iron with hepatic failure, diabetes, testicular atrophy, arthritis, cardiomyopathy, peripheral neuropathy and hyper pigmentation. Copper with cardiomyopathy and goiter. The fact that none of the subject studied, had any of the mentioned disease above calls for further biochemical studies on alcohol abuse.

Duration of intake and excessive consumption of locally brewed alcohol altered significantly the trace elements profile of alcohol abusers. This led to trace elements deficiency in the case of zinc and toxicity in the case of heavy metals like copper and manganese. These trace elements though not specific markers for useful indices in chronic alcohol abuse, could offer some reliable information towards an early detection of alcohol abuse status. The CAGE score is a reliable associate of chronicity and severity of alcohol abuse. Clinicians should continue to rely on CAGE screening instrument for alcoholism and also appreciate the reliability of trace elements changes in alcohol abuse.

Conclusion: Duration of intake and excessive use of burukutu strongly associates with the deficiency of zinc and toxicity of copper manganese and iron. Abstinence therefore should continue to be the main stay of counseling.

\section{REFERENCE}

Cawte, J; Florence, M T 1989; A magnetic milieu in North Australia Ecological Magnesium. Ecology; diagnosis in individual susceptibility synergism, Therapy, Prevention advise for the community. Int. J. Biosocial Med. Res; $11: 34-56$

Chu, N; Hochberg, F H; Calne, D B; Olanow, C W. 1995; Neurotoxicity of Manganese, in: Change L. W. \& Dyer R. S. ed. Handbook of neurtoxicity. New York, Marcel Dekker;511-546.

Fenna, O; Mix, L; Schaefer, D; Gilbert, J A L. 1971; Ethanol metabolism in racial groups. Canadian Med. Assoc;105:172.

Knowles, A; Burgess, C 1984 Practical Absorption Spectrometry. New York: Chapman and Hall,

Mayfield, D; Mc Leos, G; Hall P 1974; The CAGE questionnaire. Am J. Psych; 131:1121-3

Okoye, C ; Ekpeyoung, K I 1984; Aflatoxin B (1) - Native millet beer brewed in Jos Surburbs. Trans. Roy Soc. Med Hyg:78:41-11.

Pfeiffer C C ; Mailoux R J 1988; Hypertension Heavy Metals useful actions and as a possible repository Me. Hypotheses:26:125-130.

Prasad A S 1979; Annual Review of Pharmacology and Toxicology;20:293

Royal College of Physicians. (RCP 1978); A great and growing evil: The medical consequences of alcohol abuse. London.

Sanderson, P; 1978; Disturbance of electrolyte, water and acid-base. Medicine $3^{\text {rd }}$ Series; 11:565-570.

Schukit, M A 1998: Alcohol and Alcoholism in: Harrison's Principles of Internal Medicine $14^{\text {th }}$ Ed New York, McGraw-Hill ;2503-2508.

Srinivasan, D P 1984; Trace elements in psychiatric illness. British Journal of Hospital Medicine; 32(2):77-9.

Stanley, PC; Wakwe VC Toxic trace elements in the mentally ill patients; Post graduate medical journal. 